thin lens could be placed to mimic the whole optical system.) In our eyes, the nodal point is close to the eye's centre of rotation, and this means that when we rotate our eyes all objects in a straight line stay in a straight line, whatever their distance. But if the nodal point is a long way in front of the centre of rotation, two objects that start off in a straight line become separated when the chameleon rotates its eye (Fig. 2b). For an animal that stays camouflaged and still, and hunts monocularly, the small image displacements that eye movements produce may allow foliage at different distances to be distinguished and, along with the leaves, the creatures that live on them.

Michael F. Land is at the Sussex Centre for Neuroscience, School of Biological Sciences, University of Sussex, Brighton BN1 9QG, UK.

\title{
Life at the margins
}

\section{James $P$. Ferris}

WHAT led to life on Earth? So great a question can only be tackled by breaking it down into sections. On page 683 of this issue a group led by Stanley Miller ${ }^{1}$ does just that, by looking at the possible prebiotic synthesis of the coenzyme pantetheine. Their experiments show that this biologically active molecule could plausibly have formed in the sunbaked sludge left at the margins of drying pools and lagoons.

In modern life-forms, coenzymes are small molecules which in association with enzymes catalyse redox and group transfer reactions. Their structures vary widely, in marked contrast to those of the repetitive structural units present in proteins and nucleic acids. Many contain nucleotides as structural units including nicotinamide adenine dinucleotide $\left(\mathrm{NAD}^{+}\right)$or flavin adenine dinucleotide (FAD) but some, such as biotin or lipoic acid, bear no resemblance to nucleic acids.

There are at least two views of the prebiotic synthesis of coenzymes. One is that at an early stage of evolution they were part of the covalent structure of RNA, rather than standing alone. They assisted in an RNA-based metabolism (now called the RNA world) before the evolution of protein catalysis ${ }^{2}$. With the advent of the protein-DNA world, proteins which could bind coenzymes were produced so that the metabolic processes they catalysed could continue.

A second view is that the structural units present in coenzymes were formed independently, in chemical processes and yields comparable to those that resulted in the synthesis of nucleotides and amino acids. This hypothesis is supported by the observation that many coenzymes contain nucleotide units or appear to have been derived from nucleotides. From this it is concluded that coenzymes formed because their precursors were abundant on the primitive Earth. In this view, ribozymes (RNA enzymes) evolved which could bind these useful structures and use them in metabolic processes in the RNA world. This view is supported by the reports that RNAs have been produced by directed evolution in the laboratory which bind $\mathrm{NAD}^{+}, \mathrm{FAD}$, nicotinamide mononucleotide and adenosine triphosphate $e^{3,4}$, although these complexes don't, at present, show catalytic activity.

Here, Keefe et al. ${ }^{1}$ base their 'prebiotic' synthesis of pantetheine on the second view, the presumed abundance of its component molecules, pantoyl lactone, $\beta$ alanine and cysteamine on the primitive Earth. Modest heating $\left(40-118^{\circ} \mathrm{C}\right)$ of a dried mixture of these three substances gives pantetheine in up to 1 per cent yield, in a day to more than six weeks depending on temperature. The authors note that all three pantetheine precursors are very water soluble, and they propose that these species would be among the last to precipitate when a pond or lagoon dried out and the residue of organics remaining was further heated. Consequently, the molecules would be in close proximity so that these solid-state reactions would occur. The authors do not report the products of the reaction mixture in which pantothenic acid was used in place of pantoyl lactone, but one would expect that cyclization of the hydroxyacid to the lactone would occur ${ }^{5}$ under the dry heating conditions used, and the lactone would initiate the synthesis of pantetheine.

Keefe et al. then attempted to convert pantetheine to the nucleotide derivative dephosphocoenzyme A by heating it with ATP or ADP. But this proved unsuccessful. Phosphodiester bonds are not formed readily from di- or triphosphates under prebiotic conditions, a result which suggests either that phosphodiester groups were rare on the primitive Earth or that they formed using phosphate activating groups other than the pyrophosphate group.

This research is one of a very few papers dealing with the prebiotic synthesis of coenzymes. Proposed prebiotic syntheses of nicotinamide and its derivatives were reported $^{6,7}$ in the early $1970 \mathrm{~s}$ and the formation of $\mathrm{NAD}^{+}$from the correspond- ing nucleotides was reported ${ }^{8}$ in 1978 , but the syntheses bear little relation to the current one by Miller's group.

Of course, the synthesis of coenzymes from their components is only part of the story. How did the precursors themselves arise on the early Earth? Miller and co-workers have given this matter their attention on previous occasions, and have suggested prebiotic syntheses for cysteamine and pantoic acid, the starting materials for the formation of pantetheine and pantothenic $\operatorname{acid}^{9,10}$. Cysteamine is formed by the reaction of ethylene sulphide with ammonia. The prebiotic synthesis of ethylene sulphide has not been demonstrated but it is proposed to have been formed by the reaction of sulphur atoms with ethylene. The precursor to pantoyl lactone, pantoic acid, is formed by the reaction of formaldehyde and $\mathrm{HCN}$ with isobutyraldehyde. It is assumed that isobutyraldehyde is formed by passing an electric discharge through methane, nitrogen, ammonia and water, as the corresponding amino acid, valine, is observed as a product.

It is surprising that there have been so few reports of the prebiotic syntheses of coenzymes, as they are often assumed to have been present on the primitive Earth. Perhaps, contrary to the evidence presented in this report, most coenzymes were not synthesized readily from their component parts on the primitive Earth but are the products of an early form of life. Another possible explanation is that as most coenzymes contain a nucleotide link, more data concerning the prebiotic synthesis of nucleotides is required before we can begin to understand the prebiotic formation of coenzymes. Or perhaps the reason is simply that, as most scientists in the field of the origins of life are currently investing their time in the synthesis of oligonucleotides and oligopeptides, few have seriously addressed the question of the prebiotic formation of coenzymes. I would suspect the latter - but surely these exotic molecules deserve more attention.

James P. Ferris is in the Department of Chemistry, Rensselaer Polytechnic Institute, Troy, New York 12180, USA.

1. Keefe, A. D., Newton, G. L. \& Miller, S. L. Nature 373 683-685(1995).

White H. B molec Evol 7 101-104 (1976)

3. Burgstaller, P. \& Famulok, M. Angew. Chem. Int. Edn Engl. 33, 1084-1086 (1994)

4. Sassanfar, M. \& Szostok, J. W. Nature 264, 550-552 (1993)

5. Fridman, Y. D. et al. Russ. J. inorg. Chem. 30, 16-19 (1985).

6. Dowler, M.J. etal Science 169.1320-1321 (1970)

7. Friedmann, N. et al. Science 171, 1026-1027 (1971)

8. Lohrmann, R. \& Orgel, L. E. J. molec. Evol. 11, 17-23 (1978)

9. Miller, S. L. \& Schlesinger, G. J. molec. Evol. 36. 302-307 (1993).

10. Miller, S. L.\& Schlesinger, G. J. molec. Evol. 36. 308-314 (1993). 